\section{INVESTIGATING IMMUNE MEDIATED MECHANISMS OF PARPI RESISTANCE IN BRCA1-ASSOCIATED TRIPLE NEGATIVE BREAST CANCER (TNBC)}

${ }^{1}$ Anita Mehta, ${ }^{1}$ Madeline Townsend, ${ }^{1}$ Madisson Oliwa, ${ }^{2}$ Patrice Lee, ${ }^{2}$ Nicholas Saccomano, ${ }^{3}$ Filipa Lynce, ${ }^{3}$ Geoffrey Shapiro, 'Elizabeth Mittendorf, 'Jennifer Guerriero*. ${ }^{1}$ Brigham and Women's Hospital, Boston, MA, USA; ${ }^{2}$ Pfizer, Boulder, CO, USA; ${ }^{3}$ Dana-Farber Cancer Institute, Boston, MA, USA

Background Poly(ADP-ribose) polymerase inhibitors (PARPi) have improved the outcomes of BRCA-associated breast cancer; however, treatment responses are often not durable. Our preclinical studies demonstrated that PARPi activates the cGAS/STING pathway and recruitment of anti-tumor CD8+ T-cells that are required for tumor clearance [1]. These studies contributed to development of clinical trials testing PARPi plus immune checkpoint blockade (ICB). Unfortunately, early phase trials of PARPi + ICB have not yet suggested efficacy will be superior to PARPi monotherapy. Lack of demonstrated clinical synergy between PARPi + ICB underscores the need to study the tumor microenvironment (TME) during PARPi therapy to identify optimal strategies to enhance T-cell activation. We recently showed that PARPi induces CSF-1R+ suppressive tumor associated macrophages (TAMs) that restrict antitumor immune responses, contributing to PARPi resistance [2]. Removing TAMs with anti-CSF-1R therapy in combination with PARPi significantly enhanced overall survival (OS) compared to PARPi monotherapy in preclinical models [2]. Here, we investigate how modulating TAMs can enhance PARPi + ICB.

Methods Mice bearing BRCA1-deficient TNBC (K14-Cre; Brca1f/f;p53f/f) tumors were treated for 98 days with PARPi (Talazoparib) \pm small molecule inhibitor of CSF-1R (ARRAY382; CSF-1Ri) \pm anti-PD-1 and then followed for survival. Flow cytometry was employed to elucidate changes in the TME after treatment.

Results PARPi conferred a significant survival advantage over vehicle treated mice (median OS 33 v. 14 days; $\mathrm{p}=0.0034$ ) and 2/8 PARPi-treated mice experienced complete tumor clearance at day 98. PARPi + CSF-1Ri treated mice (median OS 140 days) remarkably cleared $7 / 10$ tumors by day 98. The addition of anti-PD-1 to PARPi did not enhance OS compared to PARPi monotherapy. The triple combination of anti-PD-1 + PARPi + CSF-1Ri has not yet significantly enhanced the median OS compared to PARPi + CSF-1Ri (ongoing; $168 \mathrm{v}$. 140 days); nor did it increase clearance of tumor by day 98 (7/10). However, the triple combination led to superior long term tumor clearance. At day 161 the triple combination exhibited 5/10 tumor free mice compared to $2 / 10$ treated with PARPi + CSF-1Ri. To elucidate how CSR-1Ri enhanced PARPi + ICB responses, flow cytometry was performed and revealed increased expression of the co-stimulatory molecule CD80, reduced tissue resident macrophages $(\mathrm{CX} 3 \mathrm{CR} 1+)$ and lower CSF-1R expression compared to PARPi + ICB.

Conclusions These data suggest that targeting immunosuppressive macrophages may induce a favorable anti-tumor immune response and enhance responses to PARPi plus ICB. We are currently evaluating the adaptive immune response in this context.

\section{REFERENCES}

1. Pantelidou, C., et al., PARP inhibitor efficacy depends on CD8+ T cell recruitment via intratumoral STING pathway activation in BRCA-deficient models of triple-negative breast cancer. Cancer Discovery, 2019: p. CD-18-1218.
2. Mehta, A.K., et al., Targeting immunosuppressive macrophages overcomes PARP inhibitor resistance in BRCA1-associated triple-negative breast cancer. Nat Cancer, 2021. 2(1): p. 66-82.

http://dx.doi.org/10.1136/jitc-2021-SITC2021.614 